\title{
Bone regeneration by human dental pulp stem cells using a helioxanthin derivative and cell-sheet technology
}

Yasuyuki Fujii ${ }^{1}$, Yoko Kawase-Koga ${ }^{1 *}$, Hironori Hojo ${ }^{2}$, Fumiko Yano ${ }^{3}$, Marika Sato ${ }^{1}$, Ung-il Chung ${ }^{2,4}$, Shinsuke Ohba ${ }^{2,4}$ and Daichi Chikazu ${ }^{1}$

\begin{abstract}
Background: Human dental pulp stem cells (DPSCs), which have the ability to differentiate into multiple lineages, were recently identified. DPSCs can be collected readily from extracted teeth and are now considered to be a type of mesenchymal stem cell with higher clonogenic and proliferative potential than bone marrow stem cells (BMSCs). Meanwhile, the treatment of severe bone defects, such as fractures, cancers, and congenital abnormalities, remains a great challenge, and novel bone regenerative techniques are highly anticipated. Several studies have previously shown that 4-(4-methoxyphenyl)pyrido[40,30:4,5]thieno[2,3-b]pyridine-2-carboxamide (TH), a helioxanthin derivative, induces osteogenic differentiation of preosteoblastic and mesenchymal cells. However, the osteogenic differentiation activities of TH have only been confirmed in some mouse cell lines. Therefore, in this study, toward the clinical use of TH in humans, we analyzed the effect of TH on the osteogenic differentiation of DPSCs, and the in-vivo osteogenesis ability of TH-induced DPSCs, taking advantage of the simple transplantation system using cellsheet technology.
\end{abstract}

Methods: DPSCs were obtained from dental pulp of the wisdom teeth of five healthy patients (18-22 years old) and cultured in regular medium and osteogenic medium with or without $\mathrm{TH}$. To evaluate osteogenesis of $\mathrm{TH}-$ induced DPSCs in vivo, we transplanted DPSC sheets into mouse calvaria defects.

Results: We demonstrated that osteogenic conditions with TH induce the osteogenic differentiation of DPSCs more efficiently than those without TH and those with bone morphogenetic protein-2. However, regular medium with TH did not induce the osteogenic differentiation of DPSCs. TH induced osteogenesis in both DPSCs and BMSCs, although the gene expression pattern in DPSCs differed from that in BMSCs up to 14 days after induction with TH. Furthermore, we succeeded in bone regeneration in vivo using DPSC sheets with TH treatment, without using any scaffolds or growth factors.

Conclusions: Our results demonstrate that TH-induced DPSCs are a useful cell source for bone regenerative medicine, and the transplantation of DPSC sheets treated with TH is a convenient scaffold-free method of bone healing.

Keywords: Dental pulp stem cells, Bone regeneration, Cell-sheet technology, Small compound, Osteogenic differentiation

\footnotetext{
* Correspondence: kogay@tokyo-med.ac.jp

${ }^{1}$ Department of Oral and Maxillofacial Surgery, Tokyo Medical University

Hospital, 6-7-1 Nishishinjuku, Shinjuku-ku, Tokyo 160-0023, Japan

Full list of author information is available at the end of the article
} 


\section{Background}

Current cell-based therapies are in need of alternative treatment techniques that enable cells to be readily harvested. Recently, dental pulp stem cells (DPSCs) were identified, which were found to differentiate into multiple cell lineages, such as adipogenic, neurogenic, and osteogenic cells [1-3]. DPSCs are now considered to be a type of mesenchymal stem cell (MSC) and demonstrate higher clonogenic and proliferative potential than bone marrow stem cells (BMSCs). Compared with other MSCs, such as BMSCs, DPSCs are very easily isolated from extracted teeth by lowinvasive surgery without any ethical issues. Therefore, DPSCs are a promising cell source for regenerative medicine and tissue engineering.

Current treatment methods are unable to fully reconstruct bone defects that are caused by fractures, cancers, congenital abnormalities, and so forth. At present, autogenous or allogeneic bones and artificial materials are used for bone augmentation. However, there are various problems with these grafts, such as the possibility of infection or absorption as well as ethical issues. To overcome such problems, a more simple and efficient method for transplantation is urgently required. Recently, cell-sheet engineering has emerged as a cell-transplantation system that requires no scaffolds or carriers, and has been used for regenerative treatment of the cornea, heart, liver, and so forth [4-6]. Cell sheets can be easily isolated by reducing the temperature of viable cells cultured on the temperature-responsive culture dish surface, with no requirement of digestive enzymes or denaturing treatments [7]. Moreover, for cell-based therapies in regenerative medicine, optimization of the culture conditions regarding proliferation and differentiation is required. Recombinant proteins, such as bone morphogenic protein (BMP), are used clinically to induce new bone formation, and BMPs were reported to have the capacity of osteogenic induction in primary human BMSCs [8]. However, recombinant proteins are expensive and unstable. Furthermore, BMPs play important roles not only in bone formation but also in the development of cancer [9]. In contrast, several studies have shown that small chemical compounds induce bone formation. For example, statins [10], TAK-778 [11], and isoflavone derivatives [12] were reported to stimulate osteogenic differentiation, and we reported previously the helioxanthin derivative 4-(4-methoxyphenyl)pyrido[40,30:4,5] thieno[2,3-

b]pyridine-2-carboxamide $(\mathrm{TH})$ to be a small molecule that induces osteogenic differentiation of preosteoblastic cells and mesenchymal cells in vitro $[13,14]$. However, the osteogenic differentiation activities of $\mathrm{TH}$ were confirmed only in specific cell lines, such as C3H10T1/2 cells and MC3T3-E1 cells, and these effects on human primary cells remain unknown.

Based on these findings, we hypothesized that DPSCs are sufficiently responsive to $\mathrm{TH}$ for osteogenic differentiation, and the combination of cellsheet engineering and TH-induced DPSCs would be a simple and convenient method for clinical application to bone regenerative medicine. Therefore, in the present study, we analyzed the effect of $\mathrm{TH}$ on the osteogenic differentiation of DPSCs in vitro and the osteogenesis of $\mathrm{TH}$-induced DPSC sheets in vivo.

\section{Methods \\ Cell isolation and culture}

This study was performed after receiving written consent from all patients and was approved by the institutional ethics committee of the Faculty of Medicine, Tokyo Medical University, Japan (approval no. 2818). DPSCs were obtained from dental pulp of the wisdom teeth of five healthy patients (18-22 years old) at Tokyo Medical University Hospital. The dental pulp was digested in a solution containing $3 \mathrm{mg} / \mathrm{ml}$ collagenase type I (Sigma-Aldrich) for $45 \mathrm{~min}$ at $37{ }^{\circ} \mathrm{C}$, and single-cell suspensions were obtained by passing the cells through a $70-\mu \mathrm{m}$ cell strainer. Human BMSCs were obtained from Takara Shuzo (C12974). The cells were seeded onto 100$\mathrm{mm}$ dishes at a density of $1 \times 10^{5}$ cells and cultured in alpha modification of Eagle's medium ( $\alpha M E M$; Gibco/BRL) supplemented with $15 \%$ fetal bovine serum (FBS; Biowest) and 1\% penicillin/streptomycin (P/S; Wako Pure Chemical Industries). All experiments were performed with DPSCs at passage 3 (P3) or P4, and with BMSCs at P4. Cells were cultured in 12-well plates (BD, Franklin Lakes, NJ, USA) and 12-well Upcell (Cell Seed) in osteogenic conditions with or without TH. For osteogenic induction, cells were cultured in $\alpha M E M$ containing 10\% FBS, 1\% P/S, 10 nM dexamethasone (Dex; Wako Pure Chemical Industries), $10 \mathrm{mM} \quad \beta$ glycerophosphate ( $\beta$-GP; Sigma-Aldrich), and $100 \mu \mathrm{M}$ L-ascorbate-2-phosphate (AsAp; Wako Pure Chemical Industries) (osteogenic medium $(\mathrm{OM})$ ). $\mathrm{TH}$, which was provided by Takeda Chemical Industries (Osaka, Japan), was dissolved in DMSO and added to OM or regular medium (RM) (Dulbecco's modified Eagle's medium (DMEM; Gibco/BRL) supplemented with $10 \% \mathrm{FBS}$ and $1 \% \mathrm{P} / \mathrm{S}$ ). For osteogenic induction with $\mathrm{BMP}$, recombinant human bone morphogenic protein 2 (rhBMP-2; Peprotech) was added to the OM (300 ng/ml). 


\section{Flow cytometry analysis}

Cells isolated from cultured DPSCs were suspended with $0.25 \%$ trypsin, washed with phosphate-buffered saline (PBS), triturated into single cells, and filtered through a $70-\mu \mathrm{m}$ cell strainer. After suspension, cells were blocked with $10 \%$ FBS for $10 \mathrm{~min}$ at $37{ }^{\circ} \mathrm{C}$, and incubated with fluorescein isothiocyanate (FITC)-conjugated antihuman CD14, FITC-human CD34, FITC-human CD44, FITC-human CD81, phycoerythrin (PE)-conjugated human CD90, and FITC-human CD105 antibodies (BioLegend) for $90 \mathrm{~min}$ at $4{ }^{\circ} \mathrm{C}$. Cells were then washed, and fixed in $4 \%$ paraformaldehyde (PFA) for $10 \mathrm{~min}$ at $4{ }^{\circ} \mathrm{C}$. Cells that were not treated with fluorescent antibodies were used as controls. The cell surface expression of markers was assessed using a FACS Verse flow cytometer (BD Biosciences). Data were analyzed using the FACS software (FlowJo; FlowJo, LLC).

\section{Alizarin Red S staining}

Cells were rinsed twice with $\mathrm{Ca}^{2+}$-free PBS and fixed in $10 \%$ formaldehyde in PBS for 10 min at $4{ }^{\circ} \mathrm{C}$. After two washes with distilled water, the cells were stained in $1 \%$ Alizarin Red S (Sigma-Aldrich) solution for $15 \mathrm{~min}$ at room temperature. The remaining dye was washed out by two washes with distilled water.

\section{Alkaline phosphatase staining}

Alkaline phosphatase (ALP) staining was performed as described previously [15]. Briefly, the cells were rinsed with PBS, fixed in 70\% ethanol, and stained for $10 \mathrm{~min}$ with $0.01 \%$ naphthol AS-MX phosphate (Sigma-Aldrich), using 1\% N,N-dimethyl formamide (Wako Pure Chemical Industries) as the substrate and $0.06 \%$ Fast BB salt (Sigma-Aldrich) as a coupler.

\section{Cell proliferation and viability}

Cell proliferation was analyzed using Cell Counting Kit8 (Dojindo). Cells were seeded at $1 \times 10^{3}$ cells per well in 96-well plates and cultured with or without TH $\left(10^{-8}\right.$ $10^{-5} \mathrm{M}$ ). After $72 \mathrm{~h}$ the labeling mixture was added to the cells, and cells were incubated for $2 \mathrm{~h}$ in a $37{ }^{\circ} \mathrm{C} / 5 \%$ $\mathrm{CO}_{2}$ incubator. The spectrophotometric absorbance of the samples was measured using a microtiter plate reader at a wavelength of $450 \mathrm{~nm}$.

\section{Real-time polymerase chain reaction analysis}

Total RNA was extracted using Trizol (Invitrogen) and reverse transcription was performed using the QuantiTect Reverse Transcription kit (Qiagen) according to the manufacturer's instructions. Realtime polymerase chain reaction (RT-PCR) was performed in a Light Cycler 96 (Roche Diagnostics) using THUNDERBIRD SYBR qPCR Mix (Toyobo) under the following conditions: $95{ }^{\circ} \mathrm{C}$ for $60 \mathrm{sec}$ and then 45 PCR cycles at $95{ }^{\circ} \mathrm{C}$ for $10 \mathrm{sec}, 65{ }^{\circ} \mathrm{C}$ for $30 \mathrm{sec}$, and $72{ }^{\circ} \mathrm{C}$ for $45 \mathrm{sec}$. To normalize for differences in the amount of total RNA added to each reaction, GAPDH was used as the endogenous control. The primer sequences used are presented in Table 1.

\section{Transplantation of DPSC sheets}

Animal experiments were performed according to a protocol approved by the Animal Care and Use committee of the Faculty of Medicine, Tokyo Medical University. Six to eight-week-old male NOD.CB17-Prkdc ${ }^{\text {scid } / J ~(N O D ~ S C I D) ~ m i c e ~(O r i e n t a l ~}$ Yeast) were used. Five mice were used for each experimental group. Each mouse was anesthetized with medetomidine $(0.75 \mathrm{mg} / \mathrm{kg})$, midazolam (4.0 $\mathrm{mg} / \mathrm{kg})$, and butorphanol tartrate $(5.0 \mathrm{mg} / \mathrm{ml})$ via intraperitoneal injection. The skin and the subcutaneous layer were incised, and the calvaria were exposed. Nonhealing and critical-sized (diameter $3.5 \mathrm{~mm}$ ) calvarial defects were created in the left parietal bone using 3.5-mm disposable biopsy punches (Kai Corporation) [16]. One defect per animal was created. DPSCs were cultured on temperature-responsive dishes in OM with or without $\mathrm{TH}$ treatment for 14 days. DPSC sheets were harvested by incubation at room temperature for $30 \mathrm{~min}$, and transplanted into these defects. The skin and subcutaneous layer were then closed by 60 nylon sutures. Eight weeks after surgery, calvarias were harvested from euthanized mice.

\section{Radiological analyses}

Micro-CT scanning was performed using a microfocus X-ray CT system (SMX-90CT; Shimadzu) under the following conditions: tube voltage, $90 \mathrm{kV}$; tube current, $110 \mu \mathrm{A}$; and field of view $(X Y), 10 \mathrm{~mm}$. The resolution of one CT slice was $512 \times 512$ pixels. The three-dimensional construction

Table 1 Sequence information of primers used for quantitative real-time polymerase chain reaction

\begin{tabular}{|c|c|}
\hline Gene & Primer sequences (forward and reverse, $5^{\prime}-3^{\prime}$ ) \\
\hline \multirow[t]{2}{*}{ GAPDH } & GAAGGTGAAGGTCGGAGTCA \\
\hline & GAAGATGGTGATGGGATTC \\
\hline \multirow[t]{2}{*}{ Runx2 } & CAGACCAGCAGCACTCCATA \\
\hline & CAGCGTCAACACCATCATTC \\
\hline \multirow[t]{2}{*}{ Alp } & ATGAAGGAAAAGCCAAGCAG \\
\hline & ATGGAGACATTCTCTCGTTC \\
\hline \multirow[t]{2}{*}{ Colla1 } & GTGCTAAAGGTGCCAATGGT \\
\hline & СTCCTCGCTTTCCTTCCTCT \\
\hline \multirow[t]{2}{*}{ Osteocalcin } & GGCAGCGAGGTAGTGAAGAG \\
\hline & AGCAGAGCGACACCCTAGAC \\
\hline
\end{tabular}



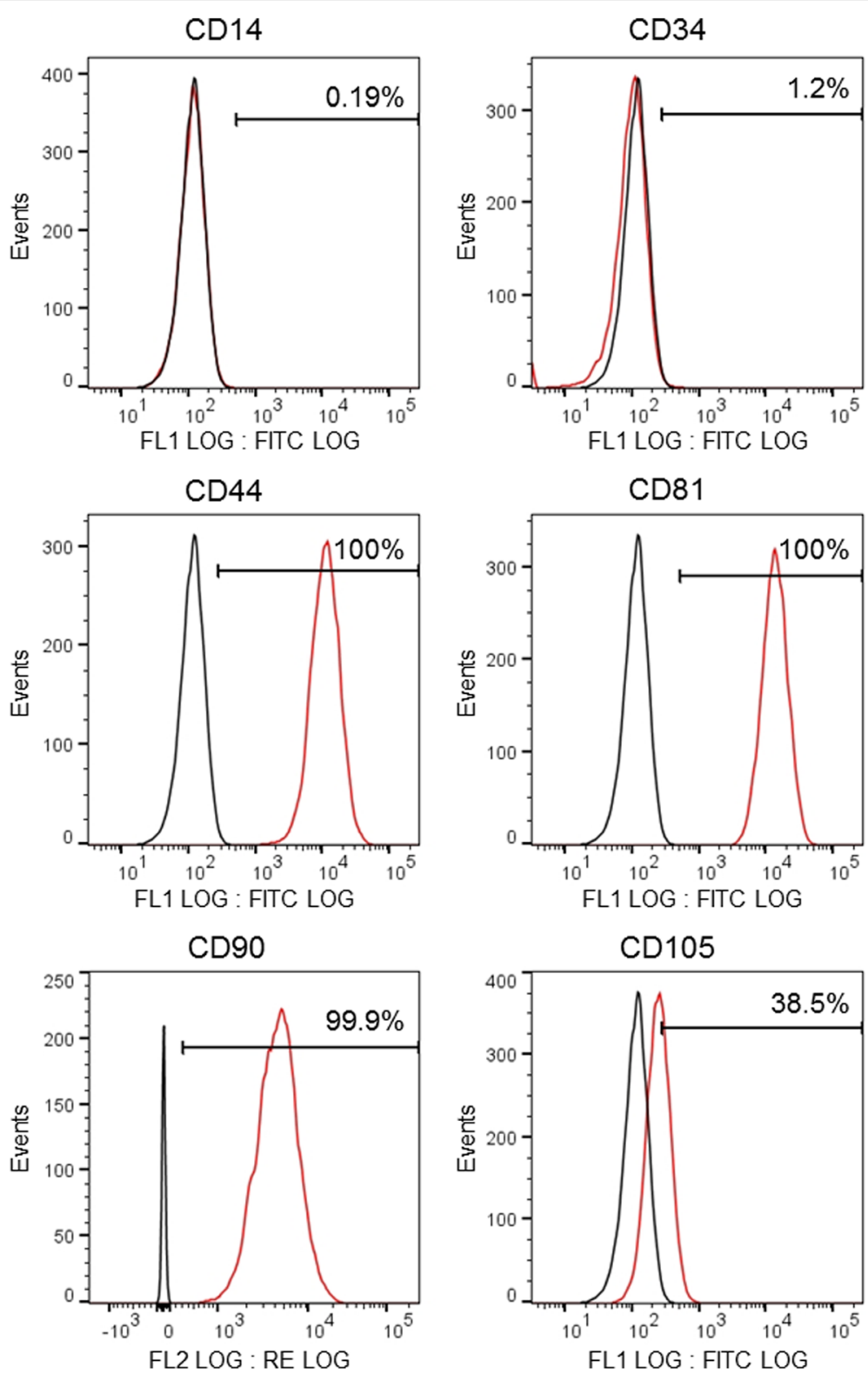

Fig. 1 Flow cytometric analysis of DPSCs. DPSCs were cultured at P3 or P4, and surface-stained with FITC-conjugated CD14, CD34, CD44, CD81, and CD105 and PE-conjugated CD90. Fluorescent signals were measured by flow cytometric analysis. Black curve represents control cells, red curve represents cells positive for these markers

software package TRI/3D-BON (Ratoc System Engineering) was used for bone morphometric analysis. Bone volume (BV), bone mineral content (BMC), and bone mineral density (BMD) were calculated within a $5 \times 5 \times 3-\mathrm{mm}^{3}$ cuboid area, in which the circular defect was adjusted to be at the center.

\section{Histological analysis}

For sectioning, mouse calvaria were fixed in $4 \%$ PFA/PBS overnight, and decalcified using 10\% ethylene- diaminetetraacetic acid (EDTA) solution under gentle shaking for 1 week. Decalcified samples were embedded in paraffin and cut into $5-\mu \mathrm{m}$ thick sections. Sections were deparaffinized and subjected to hematoxylin and eosin staining and Masson trichrome staining. Sections were observed under a microscope (BZX700; Keyence).

\section{Statistical analysis}

Data were reported as mean \pm standard deviation (SD). Statistical significance was evaluated using ANOVA and 
the Student $t$ test for comparison using SPSS 24.0 software (IBM). $p<0.05$ was considered to indicate a statistically significant difference between two groups.

\section{Results}

\section{Characterization of DPSCs}

To characterize the proportion of DPSCs under our culture conditions, we analyzed the expression of surface markers by flow cytometry. The cells were positive for CD44, CD81, CD90, and CD105, but negative for the hematopoietic stem cell makers CD14 and CD34 (Fig. 1). These data are in accordance with previous studies of DPSC surface expression $[3,17]$.

\section{Optimal concentration of TH for the osteogenic} differentiation of DPSCs

To analyze the osteogenic effect of TH in DPSCs, we cultured DPSCs in RM and OM with $\mathrm{TH}$ at concentrations ranging from $10^{-8}$ to $10^{-5} \mathrm{M}$ for 21 days. Osteogenic differentiation was evaluated by matrix mineralization visualized by Alizarin Red staining. Although $\mathrm{TH}$ induced no mineralization in DPSCs cultured in $\mathrm{RM}, \mathrm{TH}$ at $10^{-6} \mathrm{M}$ was found to induce more extensive calcification of DPSCs cultured in OM than at the other concentrations tested (Fig. 2a). TH at $10^{-5} \mathrm{M}$ suppressed matrix mineralization of DPSCs in $\mathrm{OM}$ compared with $\mathrm{TH}$ at $10^{-6} \mathrm{M}$. We also analyzed the

A

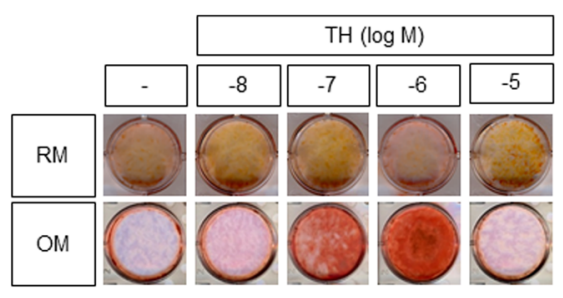

B
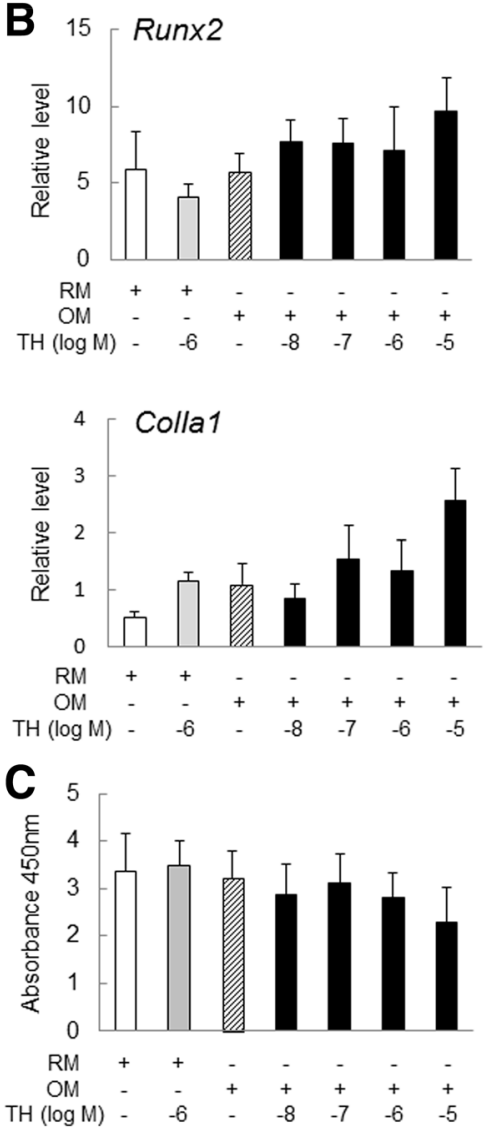
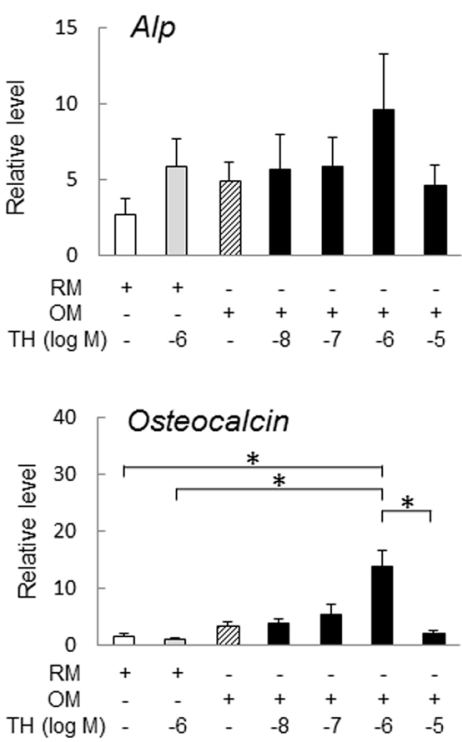

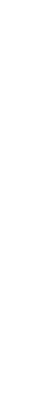


effect of various concentrations of $\mathrm{TH}$ on gene expressions of Runx2, an osteoprogenitor maker, Alp and Type I collagen alpha 1 (ColIa1), early markers of osteoblast differentiation, and osteocalcin, a mature osteoblast marker, by RT-PCR. Although there were no statistically significant differences in the expression levels of Runx2, Alp, and ColIa1, OM with TH at $10^{-6} \mathrm{M}$ significantly upregulated osteocalcin expression compared with RM alone and RM with $\mathrm{TH}$ at $10^{-6} \mathrm{M}$ (Fig. 2b). To analyze the effect of various concentrations of $\mathrm{TH}$ on the proliferation and viability of DPSCs, we used a colorimetric assay for cell proliferation. $\mathrm{TH}$ at $10^{-5} \mathrm{M}$ tended to suppress cell proliferation; however, there were no significant differences in proliferation rates between RM and $\mathrm{OM}$ with or without TH (Fig. 2c). These data suggest that $\mathrm{TH}$ induces the osteogenic differentiation of DPSCs cultured in $\mathrm{OM}$ and the optimal concentration of $\mathrm{TH}$ for the osteogenesis of DPSCs is approximately $10^{-6} \mathrm{M}$.

\section{Osteogenic differentiation of DPSCs by short-term culture with TH}

We next analyzed the effects of TH for the osteogenic differentiation of DPSCs within a short time. ALP staining showed that TH tended to upregulate and enhance ALP
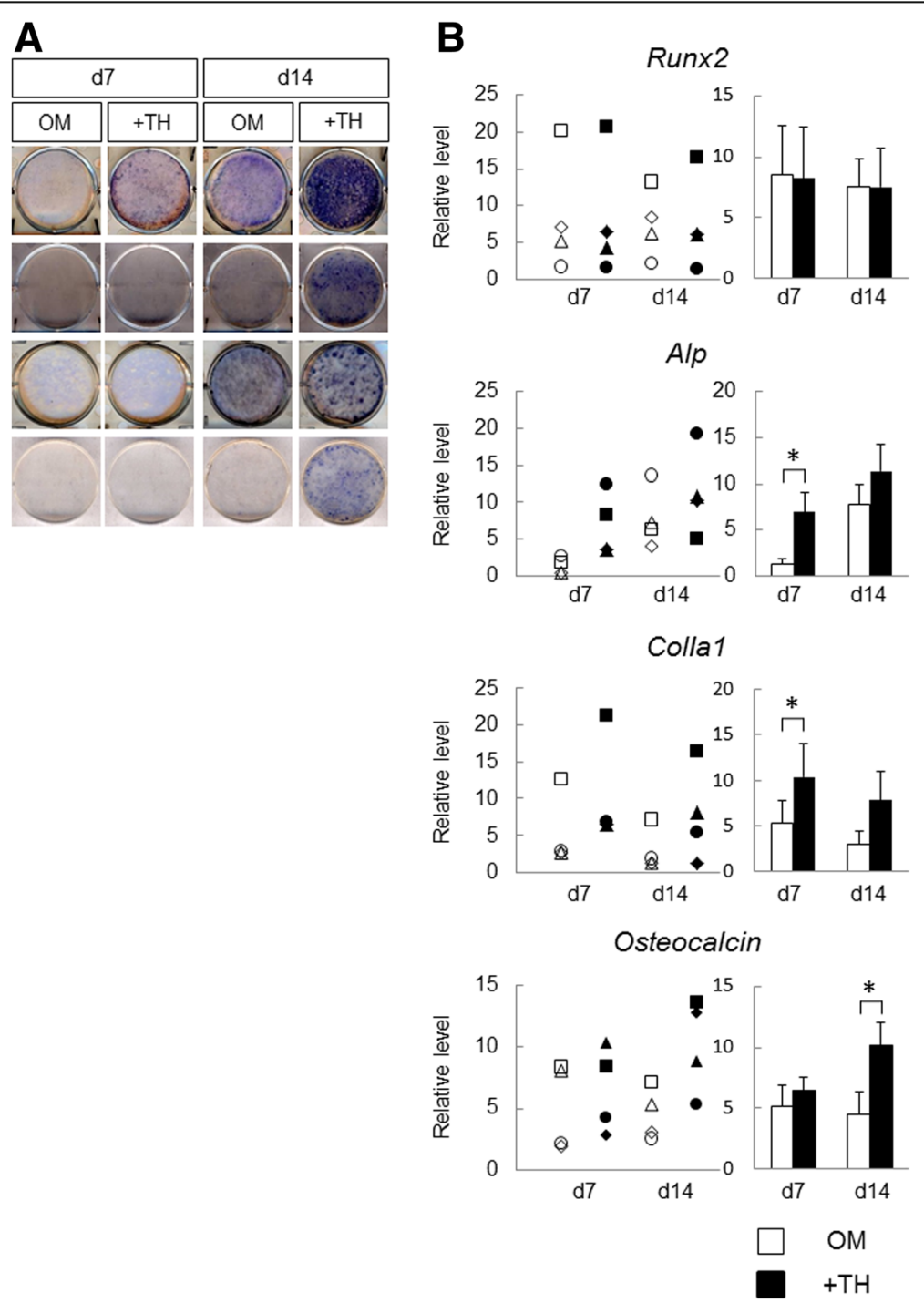

Fig. 3 Osteogenic differentiation of DPSCs with TH in short-term culture. a DPSCs cultured in OM with or without TH (10 $\left.0^{-6} \mathrm{M}\right)$. After 7 or 14 days, cells were subjected to ALP staining to detect ALP activity $(n=4)$. $\mathbf{b}$ RT-PCR performed to measure expression levels of osteogenic differentiation markers in DPSCs cultured with or without TH at day 7 or day 14. Gene expression levels of each sample plotted in the left graph, and the right graph shows the means of four samples. Error bars represent SD. Statistical significance determined by paired Student $t$ test $\left({ }^{*} p<0.05\right)$. $d$ day, OM osteogenic medium, TH 4-(4-methoxyphenyl)pyrido[40,30:4,5]thieno[2,3-b]pyridine-2-carboxamide 
activity of DPSCs at day 7 and day 14 in all samples (Fig. 3a). RT-PCR showed that TH significantly upregulated the expression of Alp and ColIalat day 7, and osteocalcin was significantly upregulated by $\mathrm{TH}$ at day 14 . However, $\mathrm{TH}$ did not induce Runx2 mRNA expression at day 7 and day 14 . These results are generally consistent with the results of ALP staining and indicate that $\mathrm{TH}$ exerts positive effects on the osteogenic differentiation of DPSCs, and its osteogenic effects begin earlier than under conventional osteogenic culture conditions.
Comparison of the osteogenic differentiation ability of DPSCs and BMSCs in the presence of TH

To demonstrate the usefulness of $\mathrm{TH}$-induced DPSCs, we compared osteogenic induction abilities between DPSCs and BMSCs in the presence of TH. ALP staining showed that TH enhanced ALP activity in both DPSCs and BMSCs at 14 days (Fig. 4a). RT-PCR analyses showed that $\mathrm{TH}$-induced BMSCs had significantly higher expression levels of Alp on day 7 and day 14 compared with TH-induced DPSCs (Fig. 4b). In contrast,

A
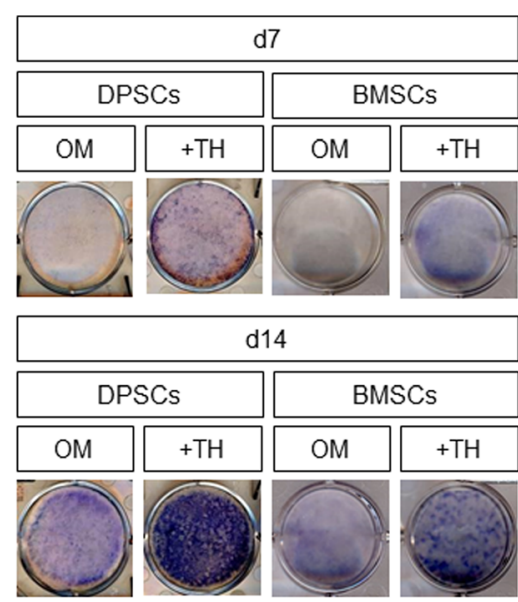

B
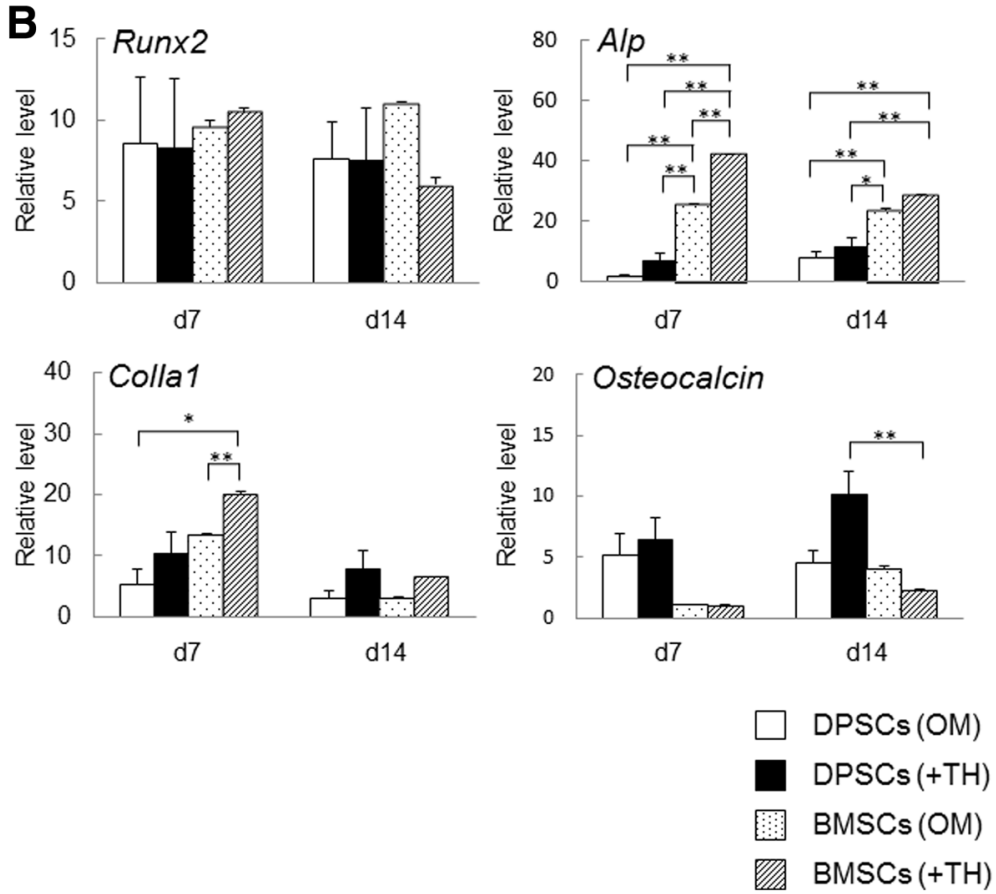

Fig. 4 Comparison of osteogenic differentiation ability of DPSCs and BMSCs in the presence of TH. a DPSCs and BMSCs cultured in OM with or without $\mathrm{TH}\left(10^{-6} \mathrm{M}\right)$. After 7 or 14 days, cells were subjected to ALP staining to detect ALP activity. $\mathbf{b}$ RT-PCR performed to measure the expression levels of osteogenic differentiation markers in DPSCs and BMSCs cultured with or without TH at day 7 or day 14 . Error bars represent SD. Statistical analyses performed by one-way ANOVA ( $\left.{ }^{*} p<0.05,{ }^{* *} p<0.01\right)$. Results of DPSCs are from four patients. Results of BMSCs are from three independent experiments. $d$ day, DPSC human dental pulp stem cell, BMSC bone marrow stem cell, OM osteogenic medium,

TH 4-(4-methoxyphenyl)pyrido[40,30:4,5]thieno[2,3-b]pyridine-2-carboxamide 
TH-induced DPSCs had significantly higher expression levels of osteocalcin on day 14 compared with $\mathrm{TH}-$ induced BMSCs. There were no statistically significant differences in the expression levels of Runx2 and ColIa1 at 14 days between $\mathrm{TH}$-induced DPSCs and $\mathrm{TH}$-induced BMSCs. These results suggest that $\mathrm{TH}$ induced osteogenesis in both DPSCs and BMSCs, although the expression pattern in DPSCs induced by $\mathrm{TH}$ was different from that in BMSCs within 14 days. Moreover, TH may more strongly promote the maturation of osteoblastic cells from DPSCs than from BMSCs.

\section{Comparison of the effect of TH and BMP-2 on the osteo- genic induction of DPSCs}

To determine the effectiveness of $\mathrm{TH}$, we compared the effect of TH and BMP-2 on the osteogenic induction of DPSCs. TH treatment more strongly enhanced the ALP activity of DPSCs compared with OM alone, whereas BMP-2 treatment did not (Fig. 5a). Although there were no statistically significant differences in the expression levels of Runx2 and Colla1 at 14 days, RT-PCR showed that $\mathrm{TH}$ significantly upregulated the expression levels of Alp and osteocalcin at day 14 compared with BMP-2 (Fig. 5b). Furthermore, there was no significant difference in the expression levels of these genes between $\mathrm{OM}$ with BMP-2 and OM alone. These results suggest that DPSCs treated with TH more strongly induced osteogenic differentiation compared with BMP-2.

\section{In-vivo bone regeneration after transplantation of TH- induced DPSC sheets}

To analyze bone regeneration by TH-induced DPSCs, we cultured DPSC sheets in 12-well temperatureresponsive dishes $\left(3.5 \mathrm{~cm}^{2} /\right.$ well $)$ with $\mathrm{TH}$ or without $\mathrm{TH}$ (control) for 2 weeks, and then the sheets were folded to fit the 3.5-mm calvarial defects of mice, and were grafted into the defects (Additional file 1: Figure S1). Although minimal bone formation was observed at the edge of the defect, we previously observed that $3.5-\mathrm{mm}$ calvarial bone defects were not covered spontaneously with
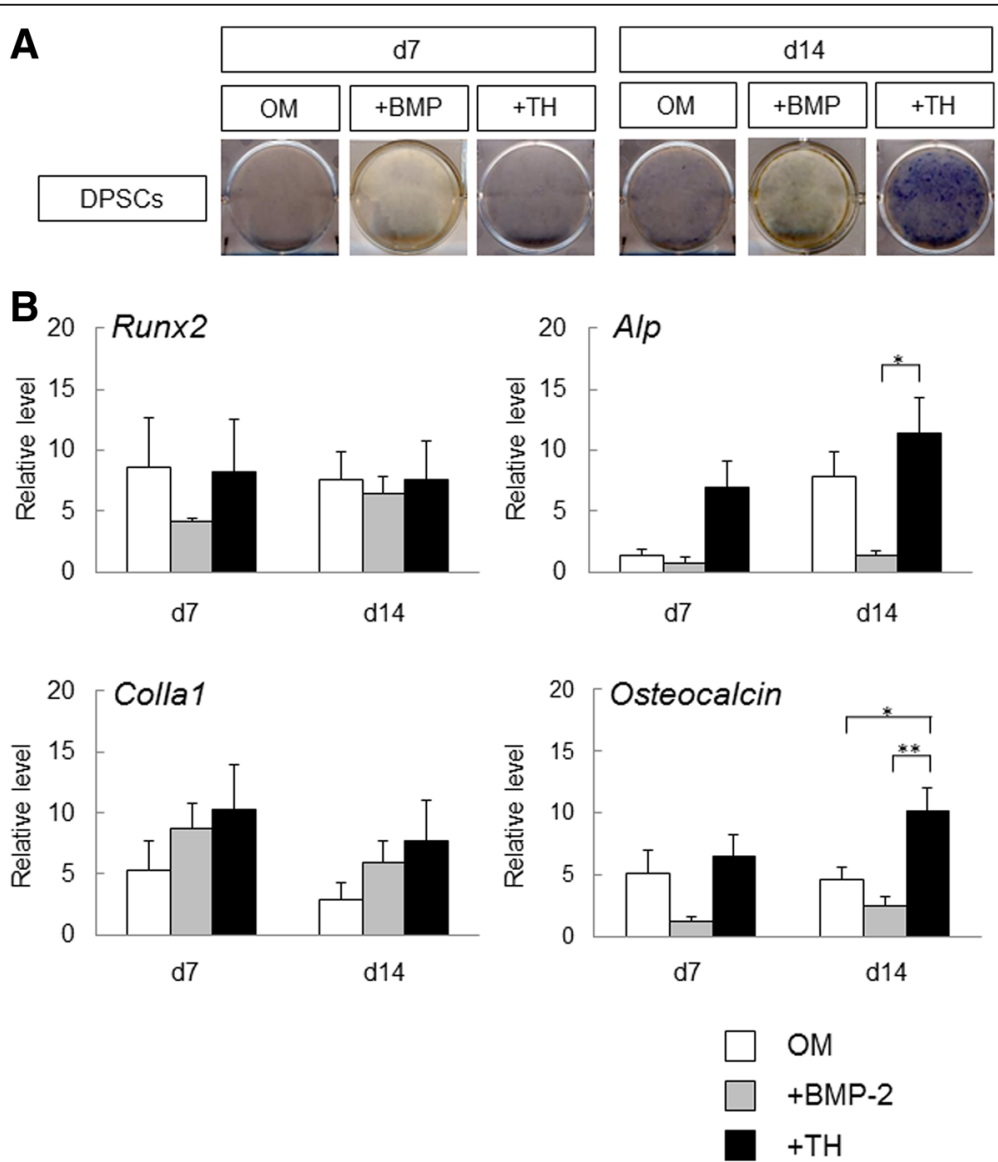

Fig. 5 Comparison of effect of TH and BMP-2 on osteogenic induction of DPSCs. a DPSCs cultured in OM with BMP-2 (300 ng/ml) or TH (10 $\left.10^{-6} \mathrm{M}\right)$. After 7 or 14 days, cells were subjected to ALP staining to detect ALP activity $(n=4)$. $\mathbf{b}$ RT-PCR performed to measure expression levels of osteogenic differentiation markers in DPSCs treated with BMP-2 or TH at day 7 or day $14(n=4)$. Error bars represent SD. Statistical analyses performed by one-way ANOVA $\left({ }^{*} p<0.05,{ }^{* *} p<0.01\right)$. d day, DPSC human dental pulp stem cell, OM osteogenic medium, TH 4-(4-methoxyphenyl)pyrido[40,30:4,5]thieno[2,3b]pyridine-2-carboxamide, BMP bone morphogenic protein 
regenerated bone within 12 weeks of surgery [18]. Therefore, we analyzed bone regeneration in the defects at 8 weeks after transplantation (Fig. 6a). Micro-CT images showed that the DPSC sheets treated with $\mathrm{TH}$ induced bone regeneration more extensively than the control sheets (Fig. 6b). Although there were no significant differences in BMD, BV and BMC of the $5 \times 5 \times 3$ $\mathrm{mm}^{3}$ cuboid areas containing the circular defects at the center were significantly increased in the TH group compared with the control group (Fig. 6c).

To investigate this further, we analyzed new bone formation by histological analyses. Hematoxylin and eosin staining showed that new bone formation was apparent in the defects treated with TH-induced DPSC-sheet compared with control defects (Fig. 7). Masson trichrome staining demonstrated that the defect area was occupied by osteoid matrix in the $\mathrm{TH}$ group. Thus, DPSC sheets treated with $\mathrm{TH}$ to induce osteogenic differentiation formed new bone tissue without requiring scaffold or growth factors.

\section{Discussion}

In the present study, we analyzed the osteogenic effects of TH on DPSCs and the in-vivo osteogenesis ability of TH-induced DPSCs using cell-sheet technology. We first demonstrated that the optimal concentration of $\mathrm{TH}$ on the osteogenic differentiation of DPSCs is approximately $10^{-6} \mathrm{M}$. Next, we found that TH induces the osteogenic differentiation of DPSCs after a short culture time. Furthermore, we demonstrated that $\mathrm{TH}$ induces the osteogenesis of DPSCs more strongly than BMP-2, and induces the osteogenesis of both DPSCs and BMSCs. Finally, DPSC sheets treated with $\mathrm{TH}$ enabled successful bone healing in a mouse calvarial defect model.

DPSCs demonstrate higher clonogenic and proliferative potential than BMSCs [2], and can easily be obtained from

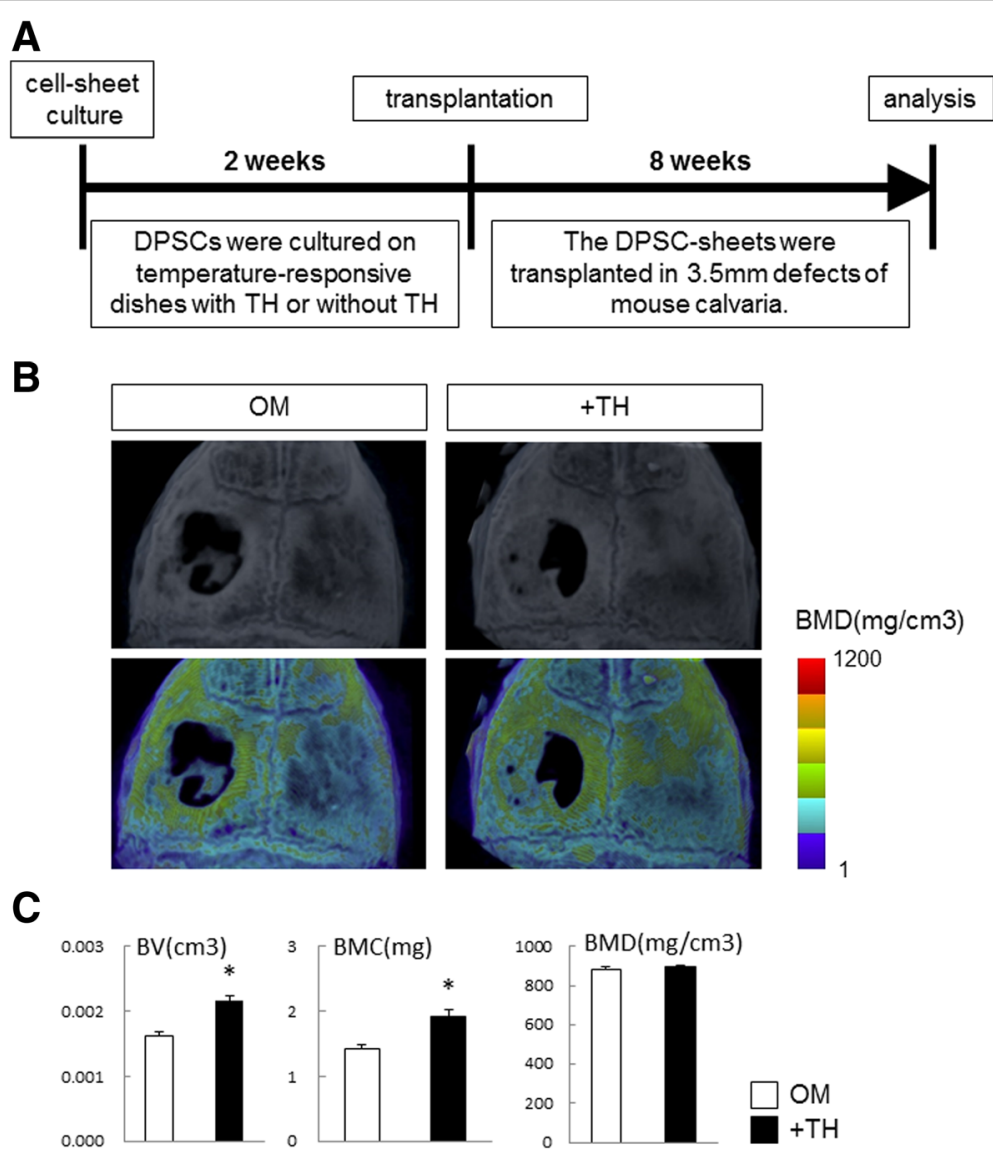

Fig. 6 Radiological findings of mouse calvarial bone defects grafted with TH-induced DPSC sheets at 8 weeks after surgery. a Experimental procedure. DPSC sheets cultured on temperature-responsive dishes in OM with or without TH $\left(10^{-6} \mathrm{M}\right)$ for 2 weeks and then transplanted into 3.5 -mm defects of mouse calvaria. At 8 weeks after transplantation, calvaria were dissected. b Micro- $C T$ images (top) and color-mapped images created from BMD values (bottom). c Quantification of BV, BMC, and BMD of $5 \times 5 \times 3-\mathrm{mm}^{3}$ cuboid samples with a circular defect in the center. Data shown as mean \pm SD of five mice per group. ${ }^{*} p<0.05$ by Student unpaired two-tailed $t$ test. DPSC human dental pulp stem cell, OM osteogenic medium, TH 4-(4-methoxyphenyl)pyrido[40,30:4,5]thieno[2,3-b]pyridine-2-carboxamide, BMD bone mineral density, BV bone volume, BMC bone mineral content 


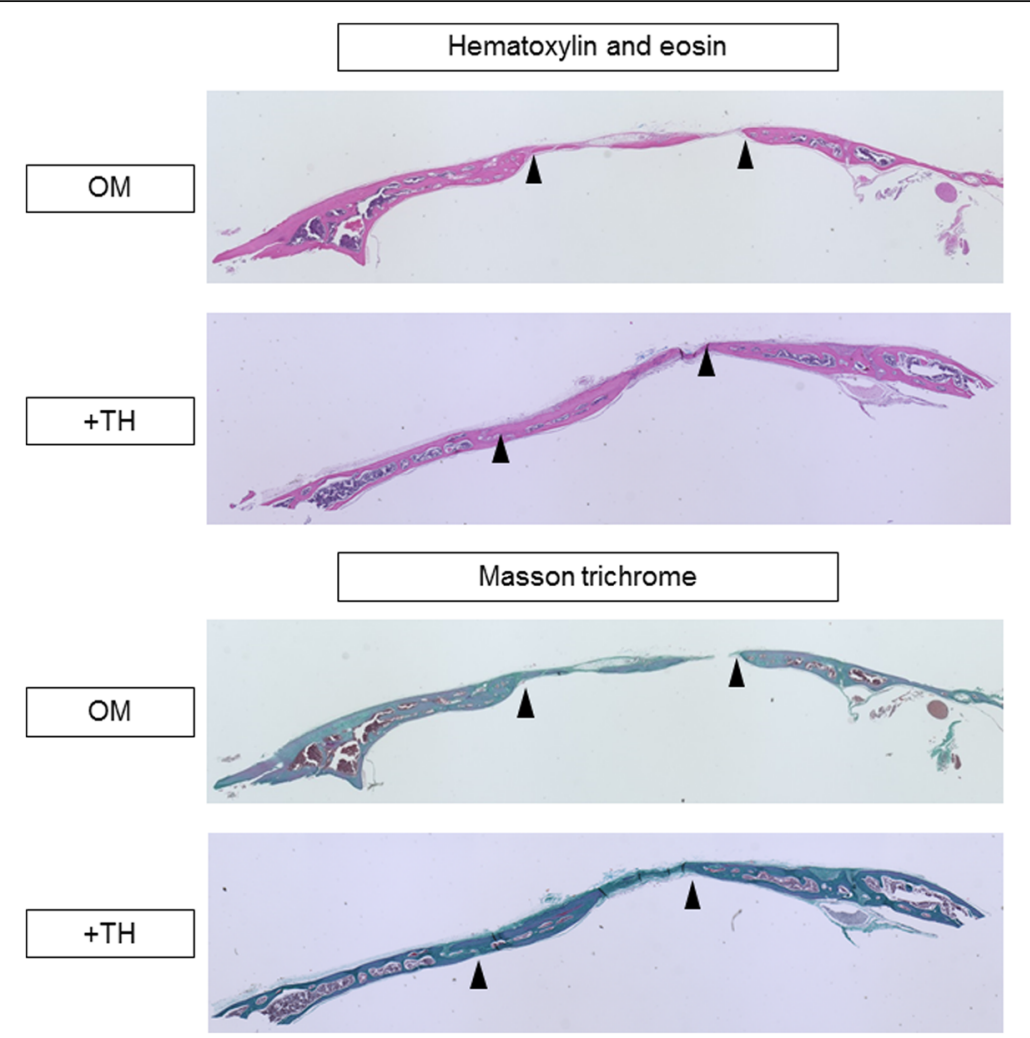

Fig. 7 Histological findings of mouse calvarial defects transplanted with DPSC sheets with or without TH treatment. Hematoxylin and eosin staining and Masson trichrome staining of mouse calvarial defects 8 weeks after transplantation of DPSC sheets, treated with or without TH (10 ${ }^{-6}$ M). Defect edges indicated by arrowheads. OM osteogenic medium, TH 4-(4-methoxyphenyl)pyrido[40,30:4,5]thieno[2,3-b]pyridine-2-carboxamide

tooth extraction surgery. DPSCs are expected to become an important cell source for regenerative medicine and tissue engineering. When using DPSCs for regenerative medicine, it is important to obtain a sufficient number of cells maintaining multipotency. However, the number of cells obtained from dental pulp is limited. Physiological secondary dentin and pathological tertiary dentin are formed by odontoblasts with age, resulting in a decrease in pulp tissue volumes [19]. In addition, MSCs progressively lose their multipotency and proliferative potential with age or during long-term culture [20]. To overcome these problems and to apply DPSCs in the clinical setting, culture methods that enable the proliferation and differentiation of DPSCs at high efficiency are required. In the present study, to develop a novel approach for bone regenerative medicine, we analyzed the osteogenic differentiation of DPSCs using $\mathrm{TH}$, which is a small osteogenic small molecule.

We reported previously that $\mathrm{TH}$ induces osteogenic differentiation of preosteoblastic cells and mesenchymal cells in vitro and in vivo [13, 14, 21], and that the optimal concentration for producing the osteogenic effects of TH on MC3T3-E1 and C3H10T1/2 cell lines is $10^{-6} \mathrm{M}$ [14]. In DPSCs, Alizarin Red staining showed that $\mathrm{TH}$ at $10^{-6} \mathrm{M}$ induced more extensive calcification than the other concentrations tested, and RT-PCR showed that $\mathrm{TH}$ at $10^{-6} \mathrm{M}$ significantly upregulated osteocalcin expression compared with the other conditions. Although $\mathrm{OM}$ with $\mathrm{TH}$ at $10^{-5} \mathrm{M}$ tended to suppress cell proliferation, there were no significant differences in proliferation rates between $\mathrm{RM}$ and $\mathrm{OM}$ with or without TH. We reported previously that $\mathrm{TH}$ at $10^{-5} \mathrm{M}$ had a lower osteogenic effect on MC3T3-E1 cells than $\mathrm{TH}$ at $10^{-6} \mathrm{M}$, probably due to the toxicity of $\mathrm{TH}$ [14]. When DPSCs were cultured in RM, TH failed to induce osteogenic induction. We also reported that the osteogenic effect of $\mathrm{TH}$ in mouse embryonic stem cells required Dex, $\beta$-GP, and AsAp [14]. These data suggest that the optimal concentration of DPSCs for osteogenesis is approximately $10^{-6} \mathrm{M}$, and that $\mathrm{OM}$ is essential for the osteogenic effects of $\mathrm{TH}$ in DPSCs.

Compared to conventional OM, TH significantly upregulated the expression levels of Alp and ColIa1 in DPSCs on day 7 of culture. Moreover, the late osteoblast marker osteocalcin was significantly upregulated by $\mathrm{TH}$ on day 14. ALP staining showed that TH treatment enabled ALP activity of DPSCs to be detected much earlier than the control in all experimental samples. For clinical application, the culture period of transplant cells should 
be minimized to prevent contamination, such as with viruses and bacteria, as well as to reduce the cost of culturing, and to use less auto serum. In this regard, we expect that $\mathrm{TH}$ will shorten the culture period for the osteogenic differentiation of DPSCs.

The properties of cell sheets from various cell sources have been analyzed previously regarding bone regeneration $[22,23]$. Because cell sheets are flexible and simple to transplantation, cell-sheet technology may replace conventional bone graft methods in the future. Our DPSC sheet transplantation method combined with $\mathrm{TH}$ achieved bone regeneration without the requirement for scaffolds or growth factors. Previous studies have shown that DPSCs have a higher ability to prevent T-cell alloreactivity and immunosuppressive activity compared with bone marrow-derived MSCs [24], and DPSC allografts enabled successful bone regeneration without requiring any immunosuppression in experimental animals [25]. Therefore, the induction of osteogenic differentiation in autologous or allogeneic DPSCs by TH may help establish a more efficient bone regeneration method, and our convenient method of DPSC sheets in combination with $\mathrm{TH}$ may overcome some of the problems associated with current bone regeneration methods, such as the invasion or stress of harvesting autologous bone and MSCs, ethical and safety issues concerned with allogeneic bone or recombinant proteins, and infection of artificial materials.

One limitation of this study is that the mechanism underlying osteogenic induction by TH in DPSCs remains unclear. We reported previously that $\mathrm{TH}$ induced the osteogenic differentiation of preosteoblasts in a BMP-dependent manner [4]. However, a combination of $\mathrm{TH}$ and BMP-2, but not $\mathrm{TH}$ alone, induced osteogenic differentiation in mouse embryonic stem cells and in mouse and human dermal fibroblasts. In contrast to the osteogenic effect of BMP-2 on BMSCs [8], in the present study BMP-2 did not induce osteogenesis in DPSCs. Furthermore, TH alone induced osteogenesis in both DPSCs and BMSCs, although the gene expression pattern induced by $\mathrm{TH}$ in DPSCs was different from that in BMSCs. The differences of the effects of TH and BMP-2 between DPSCs and BMSCs may be owing to the different origins of the cells. BMSCs are derived from the mesoderm, whereas DPSCs originate from cranial neural crest cells and express markers characteristic for cranial neural crest-derived stem cells, such as nestin, HNK-1, P75, and glial fibrillary acidic protein [26]. With regard to the osteogenic mechanisms of $\mathrm{TH}$, we are planning to investigate the direct molecular target of $\mathrm{TH}$ using a helioxanthin derivative which has a functional moiety interacting with magnetic beads through amide bonding, and hence can be purified and also retains osteogenic ability at a comparable level to that of $\mathrm{TH}$. For clinical application of
DPSCs using TH, we should analyze not only the molecular mechanisms involved in the effect of $\mathrm{TH}$ on DPSCs but also its bioactivity and toxicity.

\section{Conclusions}

We demonstrated that $\mathrm{TH}$ induces the osteogenic differentiation of DPSCs in short-term culture, leading to shortened culture duration for osteogenic differentiation of DPSCs. Furthermore, TH-induced DPSC sheets promote osteogenesis in vivo. Thus, this transplantation method of DPSCs with TH treatment may be useful for bone regenerative medicine in terms of safety and convenience.

\section{Additional file}

Additional file 1: Figure S1. Mouse calvarial defect model and procedure for implantation of DPSC sheets into a bone defect. (A) Gross appearance and micro-CT image of mouse calvarial defect model before transplantation. (B) Procedure for implanting DPSC sheets into the mouse calvarial bone defects (TIF $318 \mathrm{~kb}$ )

\section{Abbreviations \\ ALP: Alkaline phosphatase; AsAp: L-Ascorbate-2-phosphate; BMC: Bone mineral content; BMD: Bone mineral density; BMP: Bone morphogenic protein; BMSC: Bone marrow stem cell; BV: Bone volume; Colla1: Type I collagen alpha 1; Dex: Dexamethasone; DPSC: Dental pulp stem cell; EDTA: Ethylene-diaminetetraacetic acid; FITC: Fluorescein isothiocyanate; $\beta$ - GP: Beta-glycerophosphate; aMEM: Alpha modification of Eagle's medium; MSC: Mesenchymal stem cell; NOD SCID: NOD.CB17-Prkdc ${ }^{\text {scid/J; }}$ OM: Osteogenic medium; PBS: Phosphate-buffered saline; PE: Phycoerythrin; PFA: Paraformaldehyde; P/S: Penicillin/streptomycin; rhBMP-2: Recombinant human bone morphogenic protein 2; RM: Regular medium; RT-PCR: Real- time polymerase chain reaction; SD: standard deviation; TH: 4-(4- Methoxyphenyl)pyrido[40,30:4,5]thieno[2,3-b]pyridine-2-carboxamide}

\section{Acknowledgements}

The authors thank M. Kashiwagi, Y. Maeda, and members of the Ohba-Chung laboratory for their helpful input, and S. Mineo for providing technical assistance.

\section{Funding}

This work was funded by a Tokyo Medical University Research Grant, a Grantin-Aid of The Ishidsu Shun Memorial Scholarship, and Grants-in-Aid for Scientific Research C (No 16K11700) from the Ministry of Education, Culture, Sports, Science and Technology, Japan.

\section{Availability of data and materials}

All data generated or analyzed for this study are included in this published article.

\section{Authors' contributions}

YK-K, U-iC, SO, and DC conceived and designed the experiments. YF, MS, and YK-K were responsible for sample collection. YF performed the experiments and wrote the manuscript. YF, HH, FY, and $\mathrm{SO}$ analyzed the data. All authors discussed the results and commented on the manuscript. All authors read and approved the final manuscript.

\section{Ethics approval}

This study was performed after receiving written consent from all patients and was approved by the institutional ethics committee of the Faculty of Medicine, Tokyo Medical University, Japan (approval no. 2818). Animal experiments were performed according to a protocol approved by the Animal Care and Use committee of the Faculty of Medicine, Tokyo Medical University. 


\section{Consent for publication}

Not applicable.

\section{Competing interests}

The authors declare that they have no competing interests.

\section{Publisher's Note}

Springer Nature remains neutral with regard to jurisdictional claims in published maps and institutional affiliations.

\section{Author details}

${ }^{1}$ Department of Oral and Maxillofacial Surgery, Tokyo Medical University Hospital, 6-7-1 Nishishinjuku, Shinjuku-ku, Tokyo 160-0023, Japan. ${ }^{2}$ Division of Clinical Biotechnology, Graduate School of Medicine, The University of Tokyo, 7-3-1 Hongo, Bunkyo-ku, Tokyo 113-8655, Japan. ${ }^{3}$ Sensory and Motor System Medicine, Graduate School of Medicine, The University of Tokyo, 7-3-1 Hongo, Bunkyo-ku, Tokyo 113-8655, Japan. ${ }^{4}$ Department of Bioengineering, The University of Tokyo Graduate School of Engineering, 7-3-1 Hongo, Bunkyo-ku, Tokyo 113-8655, Japan.

Received: 20 September 2017 Revised: 26 December 2017 Accepted: 17 January 2018 Published online: 01 February 2018

\section{References}

1. Gronthos S, Brahim J, Li W, Fisher LW, Cherman N, Boyde A, DenBesten P, Robey PG, Shi S. Stem cell properties of human dental pulp stem cells. J Dent Res. 2002:81(8):531-5.

2. Gronthos S, Mankani M, Brahim J, Robey PG, Shi S. Postnatal human dental pulp stem cells (DPSCs) in vitro and in vivo. Proc Natl Acad Sci U S A. 2000; 97(25):13625-30

3. Tamaki $Y$, Nakahara T, Ishikawa $H$, Sato $S$. In vitro analysis of mesenchymal stem cells derived from human teeth and bone marrow. Odontology. 2013; 101(2):121-32.

4. Kawamura M, Miyagawa S, Fukushima S, Saito A, Miki K, Ito E, Sougawa N, Kawamura T, Daimon T, Shimizu T, et al. Enhanced survival of transplanted human induced pluripotent stem cell-derived cardiomyocytes by the combination of cell sheets with the pedicled omental flap technique in a porcine heart. Circulation. 2013;128(11 Suppl 1):S87-94.

5. Nishida K, Yamato M, Hayashida Y, Watanabe K, Yamamoto K, Adachi E, Nagai S, Kikuchi A, Maeda N, Watanabe H, et al. Corneal reconstruction with tissue-engineered cell sheets composed of autologous oral mucosal epithelium. N Engl J Med. 2004;351(12):1187-96.

6. Ohki T, Yamato M, Ota M, Takagi R, Murakami D, Kondo M, Sasaki R, Namiki $\mathrm{H}$, Okano T, Yamamoto M. Prevention of esophageal stricture after endoscopic submucosal dissection using tissue-engineered cell sheets. Gastroenterology. 2012;143(3):582-88. e581-2.

7. Hirose M, Kwon OH, Yamato M, Kikuchi A, Okano T. Creation of designed shape cell sheets that are noninvasively harvested and moved onto another surface. Biomacromolecules. 2000;1(3):377-81.

8. Lavery K, Swain P, Falb D, Alaoui-Ismaili MH. BMP-2/4 and BMP-6/7 differentially utilize cell surface receptors to induce osteoblastic differentiation of human bone marrow-derived mesenchymal stem cells. J Biol Chem. 2008;283(30):20948-58.

9. Miyazono K, Kamiya Y, Morikawa M. Bone morphogenetic protein receptors and signal transduction. J Biochem. 2010;147(1):35-51.

10. Mundy G, Garrett R, Harris S, Chan J, Chen D, Rossini G, Boyce B, Zhao M, Gutierrez $\mathrm{G}$. Stimulation of bone formation in vitro and in rodents by statins. Science. 1999:286(5446):1946-9.

11. Notoya K, Nagai H, Oda T, Gotoh M, Hoshino T, Muranishi H, Taketomi S, Sohda T, Makino H. Enhancement of osteogenesis in vitro and in vivo by a novel osteoblast differentiation promoting compound, TAK-778. J Pharmacol Exp Ther. 1999;290(3):1054-64.

12. Notoya K, Yoshida K, Tsukuda R, Taketomi S. Effect of ipriflavone on expression of markers characteristic of the osteoblast phenotype in rat bone marrow stromal cell culture. J Bone Miner Res. 1994:9(3):395-400.

13. Maeda Y, Hojo H, Shimohata N, Choi S, Yamamoto K, Takato T, Chung UI, Ohba S. Bone healing by sterilizable calcium phosphate tetrapods eluting osteogenic molecules. Biomaterials. 2013;34(22):5530-7.

14. Ohba S, Nakajima K, Komiyama Y, Kugimiya F, Igawa K, Itaka K, Moro T, Nakamura K, Kawaguchi H, Takato T, et al. A novel osteogenic helioxanthin- derivative acts in a BMP-dependent manner. Biochem Biophys Res Commun. 2007:357(4):854-60.

15. Ogasawara T, Kawaguchi H, Jinno S, Hoshi K, Itaka K, Takato T, Nakamura K, Okayama $\mathrm{H}$. Bone morphogenetic protein 2-induced osteoblast differentiation requires Smad-mediated down-regulation of Cdk6. Mol Cell Biol. 2004;24(15):6560-8.

16. Cowan CM, Shi YY, Aalami OO, Chou YF, Mari C, Thomas R, Quarto N, Contag CH, Wu B, Longaker MT. Adipose-derived adult stromal cells heal critical-size mouse calvarial defects. Nat Biotechnol. 2004;22(5):560-7.

17. Horibe H, Murakami M, lohara K, Hayashi Y, Takeuchi N, Takei Y, Kurita K, Nakashima M. Isolation of a stable subpopulation of mobilized dental pulp stem cells (MDPSCs) with high proliferation, migration, and regeneration potential is independent of age. PLoS One. 2014;9(5):e98553.

18. Huang KC, Yano F, Murahashi Y, Takano S, Kitaura Y, Chang SH, Soma K, Ueng SWN, Tanaka S, Ishihara K, et al. Sandwich-type PLLA-nanosheets loaded with BMP-2 induce bone regeneration in critical-sized mouse calvarial defects. Acta Biomater. 2017;59:12-20.

19. Couve E, Osorio R, Schmachtenberg O. The amazing odontoblast: activity, autophagy, and aging. J Dent Res. 2013;92(9):765-72.

20. Kern S, Eichler H, Stoeve J, Kluter H, Bieback K. Comparative analysis of mesenchymal stem cells from bone marrow, umbilical cord blood, or adipose tissue. Stem Cells (Dayton, Ohio). 2006;24(5):1294-301.

21. Nakajima K, Komiyama Y, Hojo H, Ohba S, Yano F, Nishikawa N, Ihara S, Aburatani $\mathrm{H}$, Takato $\mathrm{T}$, Chung UI. Enhancement of bone formation ex vivo and in vivo by a helioxanthin-derivative. Biochem Biophys Res Commun. 2010;395(4):502-8.

22. Kim AY, Kim Y, Lee SH, Yoon Y, Kim WH, Kweon OK. Effect of gelatin on osteogenic cell sheet formation using canine adipose-derived mesenchymal stem cells. Cell Transplant. 2017;26(1):115-23.

23. Uchiyama H, Yamato M, Sasaki R, Sekine H, Yang J, Ogiuchi H, Ando T, Okano T. In vivo $3 \mathrm{D}$ analysis with micro-computed tomography of rat calvaria bone regeneration using periosteal cell sheets fabricated on temperature-responsive culture dishes. J Tissue Eng Regen Med. 2011:5(6):483-90.

24. Pierdomenico L, Bonsi L, Calvitti M, Rondelli D, Arpinati M, Chirumbolo G, Becchetti E, Marchionni C, Alviano F, Fossati V, et al. Multipotent mesenchymal stem cells with immunosuppressive activity can be easily isolated from dental pulp. Transplantation. 2005:80(6):836-42.

25. Yamada Y, Ito K, Nakamura S, Ueda M, Nagasaka T. Promising cell-based therapy for bone regeneration using stem cells from deciduous teeth, dental pulp, and bone marrow. Cell Transplant. 2011;20(7):1003-13.

26. Yu J, Wang Y, Deng Z, Tang L, Li Y, Shi J, Jin Y. Odontogenic capability: bone marrow stromal stem cells versus dental pulp stem cells. Biol Cell. 2007:99(8):465-74.

\section{Submit your next manuscript to BioMed Central and we will help you at every step:}

- We accept pre-submission inquiries

- Our selector tool helps you to find the most relevant journal

- We provide round the clock customer support

- Convenient online submission

- Thorough peer review

- Inclusion in PubMed and all major indexing services

- Maximum visibility for your research

Submit your manuscript at www.biomedcentral.com/submit
Biomed Central 